\title{
АНАЛИЗ РАЗВИТИЯ ИННОВАЦИОННОГО ПРЕДПРИНИМАТЕЛЬСТВА РЕГИОНОВ ЦЕНТРАЛЬНОГО КАЗАХСТАНА НЕОБХОДИМОГО ДЛЯ ОЦЕНКИ ИХ КОНКУРЕНТОСПОСОБНОСТИ И ЭКОЛОГИЧЕСКИХ ФАКТОРОВ ВОЗДЕЙСТВИЯ
}

\begin{abstract}
Мингазова
Аннотация. Актуальность исследования. Развитие инновационных процессов отражает общее состояние экономики и обусловлено социально-экономическими преобразованиями. Основной контекст этих изменений связан с формированием новой, предпринимательской культурой, порядком и методами координации и взаимодействием между субъектами инновационной деятельности. Статья посвящена рассмотрению феномена инновационного предпринимательства, которое обусловлено тем, что оно относится к категории важнейших факторов устойчивого экономического развития и жизнеобеспечения любой страны, в результате чего происходит активный поиск новых парадигм, соответствующих происходящим изменениям в период интеграции и глобализации отношений.
\end{abstract}

Целью исследования является выявление факторов инновационной активности компаний, относящихся к базовым и высокотехнологичным отраслям Центрального Казахстана для разработки системы мер по повышению ее уровня.

Методы исследования. В процессе работы были применены следующие количественные и качественные методы:

- обобщение - система мер по повышению инновационной активности предпринимательства в Казахстане;

- систематизация - экономико-теоретического знания о концепциях предпринимательства;

- сравнение - на основе анализа и оценки факторов инновационной активности;

- историко-логический - с целью изучения эволюции развития инновационного предпринимательства и его роли в процессе развития экономики.

В обследовании применялись эмпирические методы исследования:

1) метод кейс-анализа, согласно которому исследование было проведено с использованием максимально возможного числа источников информации: интервью с руководителями, информация с сайтов компании, неформальное общение с сотрудниками компании, использование других источников информации;

2) на основе региональных отраслевых особенностей Центрального Казахстана дана сравнительная оценка факторов инновационной активности.

Для обработки статистических данных и анализа основных факторов, влияющих на инновационную активность в регионах Центрального Казахстана, были использованы эконометрические методы.

Информационную базу исследования составили законодательные и иные нормативные акты Республики Казахстан, статистические данные Агентства Республики Казахстан по статистике, аналитические материалы, материалы научной экономической литературы и периодической печати, материалы научно-практических конференций, данные электронных ресурсов.

Результаты исследования. Используя, приведенные статистические данные развития МСБ в РК автором была поставлена и решена задача установления такой взаимосвязи по результатам применения сложного индекса, известного как индекс структурно-динамического анализа, который известен в зарубежной литературе как инструмент «shift-share analysis». Применение метода структурно-динамического анализа для оценки конкурентоспособности экономики регионов позволил получить результаты, которые выводят в лидеры те регионы, которые располагают крупными или динамично развивающимися агломерациями, что дало основание признать значимость этой организационной формы экономического пространства для формирования критической массы конкурентоспособных отраслей.

Ключевые слова: малый и средний бизнес (МСБ), финансирование, инновации, инновационный процесс, экономическое пространство, продуктивность, конкурентоспособность отраслей.

\section{Введение}

Несмотря на сложное развитие мировой экономики в последнее время, сфера предпринимательства остается одним из ключевых механизмов активизации социально-экономической жизни общества. Развитие предпринимательства является основой экономики любой страны, так как создает благоприятные условия для оздоровления экономики: формируется и развивается конкурентная среда, преодолевается отраслевой и региональный монополизм, происходит насыщение рынка, повышается экспортный потенциал, создаются рабочие места и формируется средний класс. В казахстане поддержка именно малого и среднего бизнеса является одной из приоритетных задач государственной политики экономического развития.

В развитых экономических системах малое предпринимательство играет важную роль, обеспечивая устойчивый рост и развитие экономики, высокий уровень конкуренции, а также социальное благополучие страны. Ключевым приоритетом 
В Казахстане развитие предпринимательства является одним из приоритетных направлений экономической политики государства, и Правительство стремится к формированию среднего класса и конкурентоспособного динамичного бизнес-сообщества, ориентированного на создание новых высокотехнологичных производств с наибольшей добавленной стоимостью. И хотя показатели развития предпринимательства в Казахстане существенно отличаются от аналогичных показателей развитых стран мира, на сегодняшний день МСБ республики сумел занять соответствующую нишу в экономике государства.

В целях развития предпринимательства и улучшения бизнес-климата в Казахстане проводится системная работа по совершенствованию законодательства в сфере предпринимательства, необходимая для упрощения порядка создания и ведения бизнеса, оптимизации разрешительных систем и государственного контроля, устранения административных барьеров, реализации программы государственной поддержки предпринимательства, на основе чего были сделаны следующие выводы:

- Правительством на постоянной основе проводится системная работа по снижению административных барьеров, улучшению бизнес-климата и поддержки предпринимательства. В частности, благодаря этим мерам в Казахстане малое и среднее предпринимательство развивается относительно динамично.

- Проведена масштабная ревизия контрольно-надзорной сферы, разрешительной системы, информационных инструментов, а также всего законодательства на предмет выявления и сокращения административных барьеров, издержек бизнеса и норм, препятствующих развитию.

В Казахстане развитие предпринимательства является одним из приоритетных направлений экономической политики государства, и Правительство стремится к формированию среднего класса и конкурентоспособного динамичного бизнес-сообщества, ориентированного на создание новых высокотехнологичных производств с наибольшей добавленной стоимостью. И хотя показатели развития предпринимательства в Казахстане существенно отличаются от аналогичных показателей развитых стран мира, на сегодняшний день МСБ республики сумел занять соответствующую нишу в экономике государства.

Внедрение инноваций становится одним из приоритетных инструментов обеспечения роста государственной экономики, где инновации представляют собой эффективное средство в конкурентной борьбе, так как однозначно ведут к роту благосостояния, к привлечению неизбежных инвестиций, а также к формированию нового платежеспособного спроса.

\section{Основная часть}

В условиях глобализации значительно возрастает роль инноваций как, важного инструмента в повышении конкурентоспособности. Вопрос о возникновении инноваций, специфике развития инновационной деятельности и методов управления ею приобретает сегодня актуальнейший характер, так как уровень развития цифровых инноваций является одним из определяющих факторов конкурентоспособности национальной экономики.

Приоритет централизованных методов управления инновационными процессами доказан мировой практикой. Динамичное развитие инновационной сферы - одно из главных слагаемых инновационной экономики. Такая высокотехнологичная экономика предполагает наличие эффективной инновационной системы и создание институтов поддержки инновационного процесса [1, с.78-81].

Широкое внедрение в хозяйственный оборот инновационных продуктов и технологий стало ключевым фактором экономического развития и улучшения качества жизни населения [2, с.6].

Прежде чем рассматривать различные подходы к определению понятия предпринимательство, инновации, хотелось бы определить свое видение этих терминов. Это, в первую очередь, феномен новизны, который формируется в условиях развития общества и сферы технологического и экономического развития под влиянием модернизации общественного бытия, так как результатом инновационного развития и инноваций в целом являются общественные выгоды, повышение эффективности и социально-экономическое развитие.

Эффективность использования инновационного потенциала, как считают Кулмаганбетова А.С., Дубина И.Н., Рахметулина Ж.Б., определяется не только уровнем научных исследований и разработок, но и совокупностью соответствующих технических, производственных, организационных, маркетинговых, финансовых операций, включенных в инновационные процессы [3, с.57].

Ученые всего мира убеждены, что предпринимательство играет центральную роль в экономическом развитии, создании рабочих мест и повышении уровня жизни людей в различных сообществах (Bell, R. 2015 [4]; Karimi, S., Biemans, H. J. A., Mahdei, K. N., Lans, T., Chizari, M., \& Mulder, M. (2015) [5]; Prakash, Jain, \& Chauhan, (2015) [6]. Эта точка зрения отчасти основывается на провозглашениях таких выдающихся ученых, как Шумпетер, Шульц, Кантильон, которые выделяют, что предпринимательская деятельность является мощной движущей силой экономической активности (Moroz \& Hindle, 2012 [7]; Solesvik, Westhead, Matlay, \& Parsyak, 2013) [8].

Если предпринимательство является неотъемлемой частью социально-экономических преобразований, то логично предположить, что в обществах, где больше людей с предпринимательскими способностями имеют больше возможностей для экономического прогресса по сравнению с обществами $\mathrm{c}$ меньшей предпринимательской активностью.

Как утверждают Bobylev S.N., Solovyeva S. V., важным вопросом является выбор ключевых индикаторов, которые показывают, в каком направлении происходит развитие экономики [9, с.63]. 
Вопросы эффективности деятельности малых и средних предприятий (далее - МСП) широко рассматриваются в зарубежной литературе. Как развитые, так и развивающиеся страны сильно обеспокоены ростом и эффективностью МСП. Литература, исследующая эффективность деятельности, обширна, однако имеет определенные противоречия. Чтобы выявить причины этих противоречий, исследователями Аутап AbuRumman, Ata Al Shraah, Faisal Al-Madi, Tasneem Alfalah (2021) было проведено исследование, целью которого было определить посредническую роль динамических способностей, которые развиваются благодаря предпринимательским сетям и предпринимательской ориентации [10].

В основном, исследование направлено на изучение внутренних факторов бизнеса и их влияния на результаты деятельности фирмы в микро- и малых предприятиях. В исследовании Abriham Ebabu Engidaw (2021) использовался описательный и объяснительный исследовательский дизайн и применялся количественный исследовательский подход, где зависимой переменной является производительность МСП, а независимыми переменными - управленческие факторы, факторы рабочего места и предпринимательские факторы, результаты которых показывают, что независимые переменные управленческие факторы и факторы, связанные с рабочим местом, оказывают положительное и значительное влияние на эффективность малого бизнеса в исследуемом регионе. Кроме того, существует незначительная связь между предпринимательскими факторами и зависимой переменной [11].

Потребность в достижениях, склонность к риску и внутренний локус контроля обусловили статистически значимые различия в предпринимательских намерениях. Однако из трех психологических черт только потребность в достижении частично опосредована взаимосвязью между эффектами предпринимательского образования и предпринимательскими целеустремлениями [12].

Таким образом, несмотря на множество уже существующих механизмов и моделей, доказавших свою продуктивность в других странах, специфические особенности социально-экономического устройства нашей страны требуют разработки управления в формирования инновационной активности предприятий различных сфер деятельности.

Инновационные процессы - неотъемлемая черта современного бизнеса [13, с.51]. Широкое внедрение в хозяйственный оборот инновационных продуктов и технологий стало ключевым фактором экономического развития и улучшения качества жизни населения [14, с.6].

По словам Орлова С.Н., малый и средний бизнес способствует обеспечению экономической, социальной и политической стабильности, оперативно адаптируется к потребностям рынка, эффективно имплементирует новые технологии и научные разработки, способствует формированию конкурентной среды, росту занятости, увеличению налоговых поступлений в бюджеты всех уровней [15, с.27].

Один из ключевых вызовов для Казахстана - это усиление в глобальном масштабе конкурентной борьбы за факторы, определяющие конкурентоспособность инновационных систем, в первую очередь, за высококвалифицированную рабочую силу и «умные» деньги (инвестиции, привлекающие в проекты новые знания, технологии, компетенции), резкое повышение мобильности этих факторов. Эти вызовы диктуют необходимость опережающего развития отдельных специфичных направлений научных исследований и технологических разработок

По мнению Kirilchuk, S. P., \& Nalivaychenko, E. V. (2017) проблема поиска индикаторов инновационной деятельности в настоящее время приобрела всеобщий характер [16, с.1025].

Вопросы исследования. В настоящее время предпринимательство обрело именно те размеры, которые ему позволяет достичь современная структура экономики и иные сложившиеся по итогам реформ экономические реальности, а ее подъем на уровень выше этой «планки» может быть, достигнут в дальнейшем только посредством кардинальных социально-экономических изменений и сменой самой парадигмы развития страны и регионов.

Научная новизна. В современных научных исследованиях все больше наблюдается синтез экономической теории и пространственного анализа. Единицей анализа в новой экономической географии всегда является территория или регион, в то время как микро- и макро-уровнь не рассматривают региональный профиль изучаемого явления. 


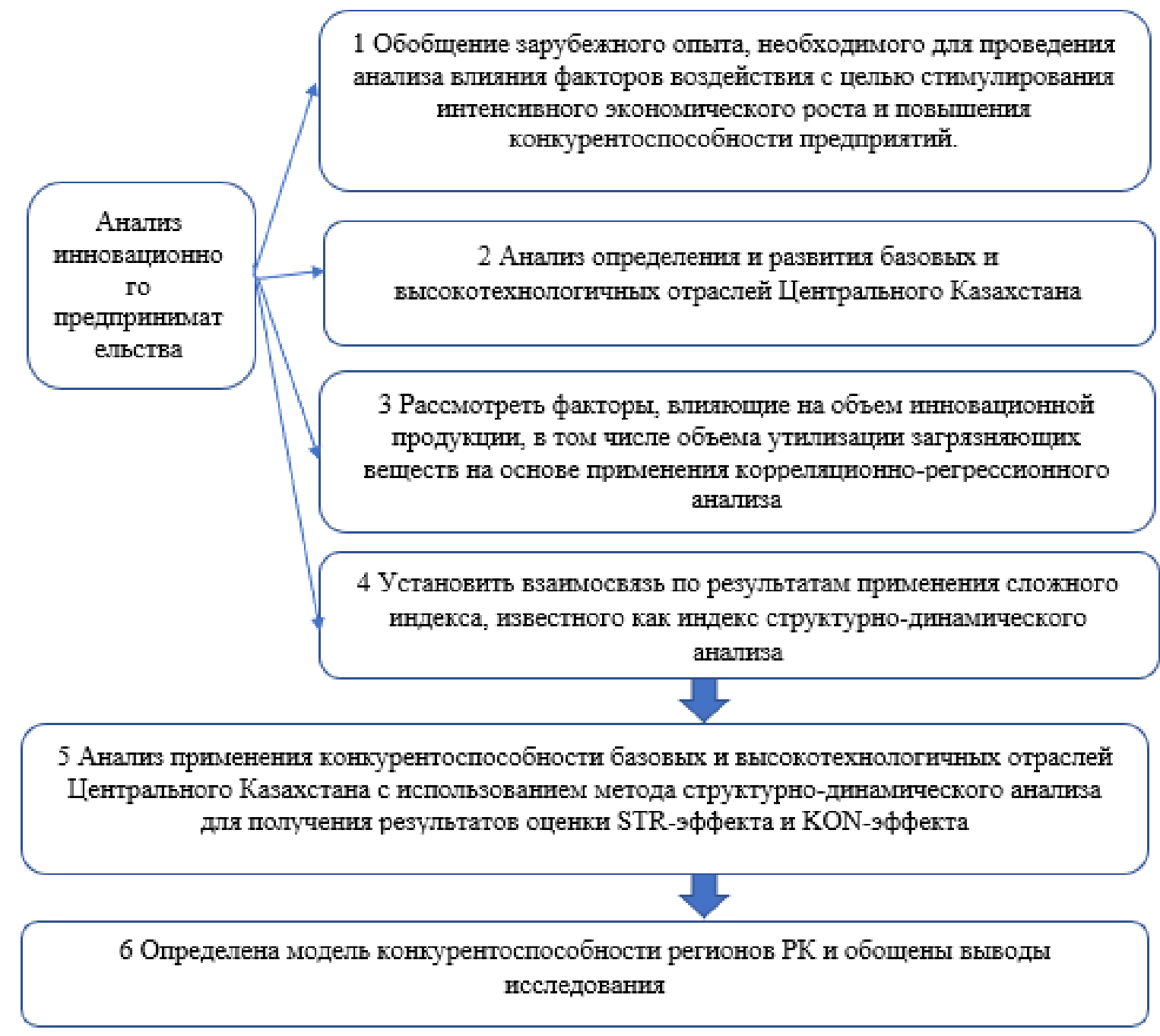

Рисунок 1 - Вопросы исследования

В связи с этим, автором была поставлена и решена задача установления такой взаимосвязи по результатам применения сложного индекса, известного как индекс структурно-динамического анализа. Этот индекс известен в зарубежной литературе как инструмент «shift-share analysis», но понимание его затруднено не только сложностью расчетов, но и трудностями объяснения. Также была предпринята попытка упрощения расчетов без потери содержания и представления их результатов в графической форме для облегчения трактовки, что ранее никем не было сделано.

Совершенствование государственной политики в сфере инновационного развития МСБ дает возможность созданию и активному внедрению инноваций в Казахстане. Социально-экономическая политика Республики Казахстан на 2017-2021 годы направлена на дальнейшую реализацию нового курса развития Республики Казахстан «Стратегии Казахстан - 2050» с учетом реализации Государственной программы «НұрлыЖол - Путь в будущее», направленной на формирование единого экономического рынка путем интеграции макрорегионов страны на основе выстраивания эффективной инфраструктуры на хабовом принципе для обеспечения долгосрочного экономического роста Казахстана, а также реализация антикризисных мер по поддержке отдельных секторов экономики в условиях ухудшения конъюнктуры на внешних рынках, и создания модели экономики Казахстана, определённой в пяти институциональных реформах Плана нации «100 конкретных шагов» [17].

23. Согласно исследованиям Ștefan Cristian Gherghina, Mihai Alexandru Botezatu, Alexandra Hosszu, Liliana Nicoleta Simionescu (2020), малые и средние предприятия (МСП) играют решающую роль в местном экономическом развитии, играя примечательную роль в создании рабочих мест, сокращении масштабов нищеты и экономическом росте [18, с.347].

Развитие инновационных процессов отражает общее состояние экономики и обусловлено социальноэкономическими преобразованиями. Основной контекст этих изменений связан с формированием новой, 
предпринимательской культурой, порядком и методами координации и взаимодействием между субъектами инновационной деятельности, где основой инновационного развития являются малые и средние предприятия, которые являются технологическими лидерами в зарождающихся отраслях экономики, а также открывают новые сегменты рынка, создают производства, способствуя формированию совершенно новых научно-технологических укладов [19, с.78].

Kazakova M.V., Pospelova E.A. утверждают, что реализация в странах СНГ национальных стратегий инновационного развития базируется на модернизации и промышленном развитии регионов, которые должны способствовать привлечению инвестиций в проекты индустриального и инновационного характера, развитию предпринимательства в научно-технической сфере [20]. Этого мнения придерживаются и другие ученые Maschenko M.V, Volkova E.A. [21], Ovsyannikova T.Yu, Rabtsevich O.V., Yugova I.V. [22]. При этом возникает интерес к оценке тенденций развития инновационной деятельности.

Вопросы развития, становления и управления инновациями приобретают сейчас повышенную актуальность, а понимание общих законов и закономерностей таких процессов является необходимым элементом для управления.

Теории и модели региональной экономики создаются для того, чтобы объяснить наблюдаемые процессы размещения хозяйствующих субъектов в экономическом пространстве, выявить тенденции и факторы конкурентоспособности отдельных территорий, объяснить различия между ними в уровне экономического развития и спрогнозировать тренды ближайшего будущего.

Систематизация методов оценки конкурентоспособности регионов не основано на признании количественной взаимосвязи между параметрами экономического пространства (плотность, размещение, связанность) и экономическим ростом отраслей, и не позволяет установить такую взаимосвязь в функциональной, либо корреляционной форме.

В связи с этим, автором была поставлена задача установления такой взаимосвязи по результатам применения сложного индекса, известного как индекс структурно-динамического анализа, который известен в зарубежной литературе как инструмент «shift-share analysis», но понимание его затруднено не только сложностью расчетов, но и трудностями объяснения.

Статистический индекс разделяется на две компоненты:

- эффект состава отраслевой структуры региона (STR -эффект); $(\mathrm{KON})$.

- темпы роста отрасли в регионе в сравнении со среднеотраслевыми национальными темпами роста отрасли

Первый - STR -эффект - определяет конкурентоспособность региона по доле отрасли в структуре региона, сложившейся под влиянием факторов внешнего и национального спроса. Удельный вес отрасли является своего рода результатом действия этих факторов за рассматриваемый период. В то же время, измеряемая разница между национальным и отраслевым темпом роста является индикатором развития отрасли, которая может расти больше или меньше, чем национальная занятость в целом.

STR эффект рассчитывается по формуле (1):

$$
\text { STRir }=\frac{E_{i r}^{0}}{E_{r}^{0}} *\left(\frac{E_{i n}^{1}}{E_{i n}^{0}}-\frac{E_{n}^{1}}{E_{n}^{0}}\right) * 100 \%,(1)
$$

Второй - KON - эффект - измеряет возможности каждого конкретного региона в превышении национального темпа роста по конкретной отрасли. Если этот прирост за период положителен, то отрасль в регионе развивается более динамично, чем в среднем по стране, что также позволяет сделать вывод о её конкурентоспособности.

KON эффект мы предлагаем рассчитывать по формуле (2):

$$
\text { KONir }=\frac{E_{i r}^{0}}{E_{r}^{0}} *\left(\frac{E_{i r}^{1}}{E_{i r}^{0}}-\frac{E_{i n}^{1}}{E_{i n}^{0}}\right) * 100 \%
$$

где:

$E_{i r}^{0}$ - объем производства в і отрасли в г регионе в базисном году;

$E_{r}^{0}$ - объем промышленного производства в r регионе в базисном году;

$E_{\text {in }}^{1}$ и $E_{\text {in }}^{0}$ - объем производства в і отрасли в стране в отчетном и базисном году;

$E_{n}^{1}$ и $E_{n}^{0}-$ бъем промоышленного производства в стране в отчетном и базисном году.

Этот подход позволяет разделять структурные факторы (STR) и краткосрочные факторы региональной конкурентоспособности (KON), определяемые динамикой роста в краткосрочном периоде.

Таким образом, можно сделать вывод, что свойства экономического пространства на современном уровне исследований конкурентоспособности регионов страны не находят отражения в методологии применяемых методов оценки, за исключением экономико-математических моделей. Большинство моделей устанавливают 
такую взаимосвязь на основе показателей экономического роста и урбанизированности экономического пространства, другие же в силу сложности задачи нами не были рассмотрены.

Обследование практики работы многих компаний и анализ теоретических исследований, проведенный нами, показал, что формирование бизнес-модели позволяет на достаточно конструктивной основе совершенствовать традиционную систему управления предприятием единичного типа, однако требует теоретического обоснования выбора характеристик и свойств управления с учетом отраслевых особенностей предприятия, его специализации.

Теории и модели региональной экономики создаются для того, чтобы объяснить наблюдаемые процессы размещения хозяйствующих субъектов и расселения населения в экономическом пространстве, выявить тенденции и факторы конкурентоспособности отдельных территорий, объяснить различия между ними в уровне экономического развития и спрогнозировать тренды ближайшего будущего.

Базовые и высокотехнологичные отрасли Центрального Казахстана оказывают существенное воздействие на экономическое развитие Республики Казахстан, которые выражаются, главным образом, в привлечении иностранных инвестиций, обеспечении экспортных и налоговых поступлений, создании значительного объема добавленной экономической стоимости и поддержке рабочих мест (вклад в формирование трудовых доходов населения) (Рисунок 2).

К базовым отраслям в Республике Казахстан относятся: угольная, металлургическая, химическая промышленность. Необходимость определения металлургии в качестве базовой отрасли промышленности исходит из того, что именно в данной отрасли производится 65\% всей промышленной продукции, заняты более 62 тыс. человек (в \% к занятости в регионе).

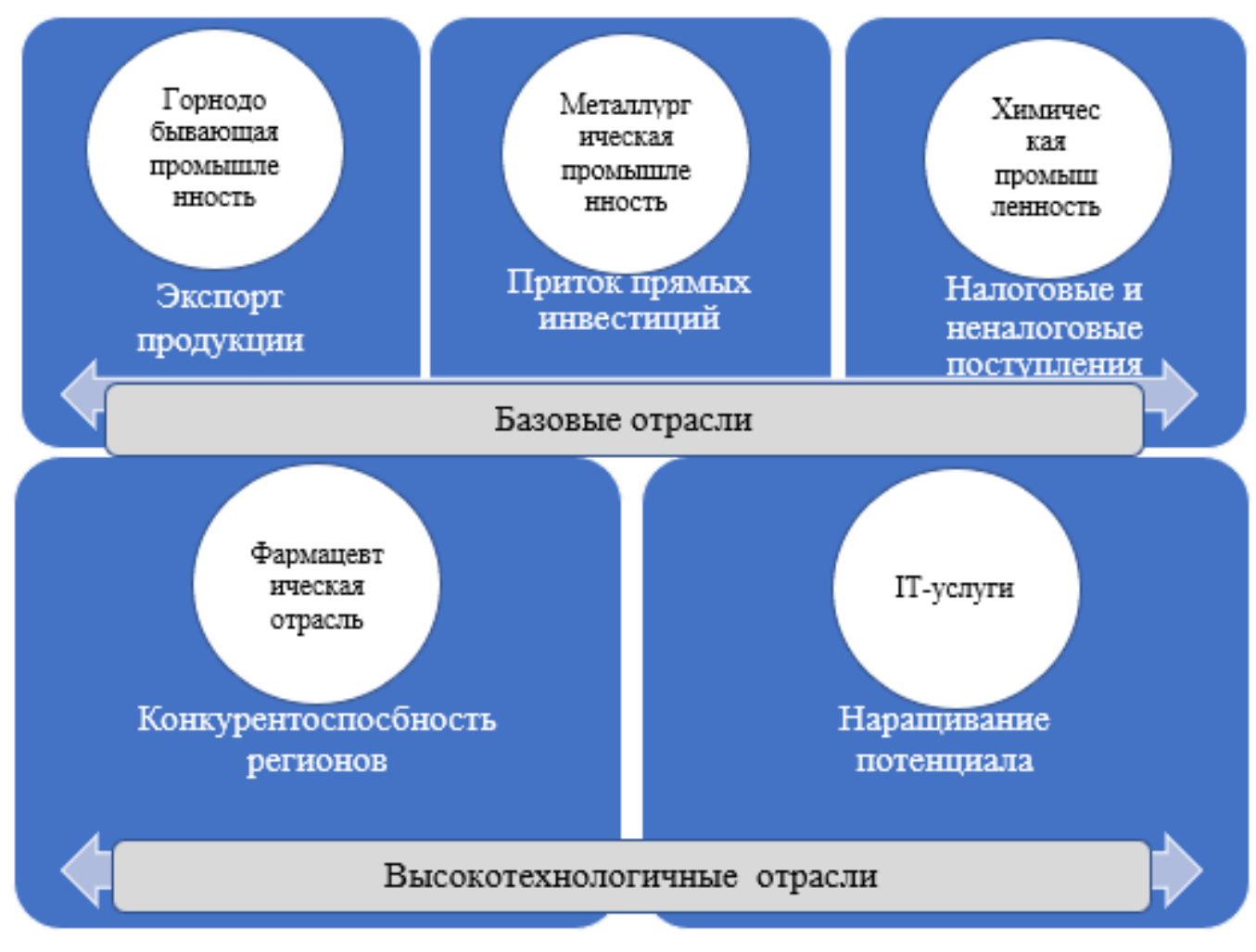

Источник: Составлен автором

Рисунок 2 - Базовые и высокотехнологичные отрасли Центрального Казахстана

Одним из показателей, с помощью которого можно судить об инновационной активности региона является объем инновационной продукции. На данный показатель оказывают влияние факторы разного характера, в том числе экономического и научно-технологического. Совокупное влияние данных факторов может быть учтено на основе корреляционно-регрессионного анализа.

В процессе анализа были рассмотрены следующие факторы: ВРП, количество инновационно-активных предприятий по всем типам инноваций, количество организаций (предприятий) осуществлявших НИОКР, внутренние затраты на НИОКР, затраты на инновации.

Для отбора факторов, которые должны быть включены в множественное линейное уравнение регрессии, были использованы статистические данные указанных показателей. 
В связи с тем, что при оценивании множественной линейной регрессии для обеспечения статистической надежности результатов требуется, чтобы число наблюдений по крайней мере в 3 раза превосходило число оцениваемых параметром, в искомую модель может быть включено только три фактора.

Анализ корреляционных матриц, построенных для трех рассматриваемых областей, показал, что в регрессионную модель необходимо включить следующие факторы: ВРП, количество инновационно-активных предприятий по всем типам инноваций, затраты на инновации. Между данными факторами отсутствует мультиколлинеарность и наблюдается значительная взаимосвязь с результирующим признаком.

Результат аппроксимации данных с помощью метода наименьших квадратов представлены в таблице 1 [23].

Таблица 1

Результаты оценки уравнений объема инновационной продукции

\begin{tabular}{|c|c|c|c|}
\hline Переменные & Карагандинская область & $\begin{array}{c}\text { Павлодарская } \\
\text { область }\end{array}$ & $\begin{array}{c}\text { Восточно-Казахстанская } \\
\text { область }\end{array}$ \\
\hline$R^{2}$ & 0,5655 & 0,6708 & 0,8420 \\
\hline Скорректированный $R^{2}$ & 0,4207 & 0,5610 & 0,7894 \\
\hline \multicolumn{4}{|c|}{ Объем инновационной продукции - зависимая переменная } \\
\hline ВРП (млн. тенге) & $0,0111 *$ & $0,1369 *$ & $0,1653 * * *$ \\
\hline $\begin{array}{c}\text { Количество инновационно- } \\
\text { активных предприятий по всем } \\
\text { типам инноваций (единиц) }\end{array}$ & $132,8199 *$ & $263,7493 *$ & $160,9918 * *$ \\
\hline Затраты на инновации (млн. тенге) & $0,7228 *$ & $0,2152 *$ & $0,1668 *$ \\
\hline \multicolumn{4}{|c|}{$* \mathrm{p}<0,1 ; * * \mathrm{p}<0,05 ; * * * \mathrm{p}<0,01$} \\
\hline
\end{tabular}

Источник: Составлена автором на основе расчетов

Анализ полученные параметров уравнения множественной линейной регрессии позволяет сделать следующие выводы:

1) Наибольший прирост объема инновационной продукции, при увеличении ВРП на 1 млн. тенге наблюдается в Восточно-Казахстанской области.

2) Наибольшее увеличение объема инновационной продукции при увеличении количества инновационноактивных предприятий на 1 единицу наблюдается в Павлодарской области.

3) Наибольшая отдача от вложений в инновации (затрат на инновации) наблюдается в Карагандинской области.

Особенностью анализа динамики и факторов инновационной активности в базовых и высокотехнологичных отраслях регионов Центрального Казахстана является то, что показатель объема произведенной продукции является показателем и инструментом экономического планирования, применяемый для оценки конкурентоспособности отдельных отраслей в региональном аспекте.

Несмотря на то, что инновационная активность в базовых и высокотехнологичных отраслях оказывает благоприятное воздействие на экономику страны, то на экологическую обстановку развитие отраслей имеет негативное воздействие. Так, например, угольная промышленность оказывает влияние не только на территорию угледобывающих предприятий, но и сказывается на окружающей среде близлежащих населенных пунктов. Что касается отвалов, образующихся при добыче угля, то даже в случае снижения добычи угля и даже прекращения разработок, на угольных разрезах возможно увеличение выбросов от уже вскрытых пластов, т.к. вступая в реакцию с водой и воздухом, они могут возгораться, увеличивая негативное воздействие на атмосферный воздух.

Некомпетентное региональное и местное планирование ухудшило ситуацию во многих местах. В ослаблении давления, оказываемого человеческой деятельностью на биоразнообразие необходимо предусмотреть мероприятия, учитывающие особенности биологии видов, находящихся в угрожаемом состоянии. Поэтому необходимы инновационные методы развития предпринимательства в базовых и высокотехнологичных отраслях экономики, направленные на утилизацию загрязняющих веществ, так как динамика за последние годы свидетельствует о недостаточном объеме утилизации (Рисунок 3) [24]. 


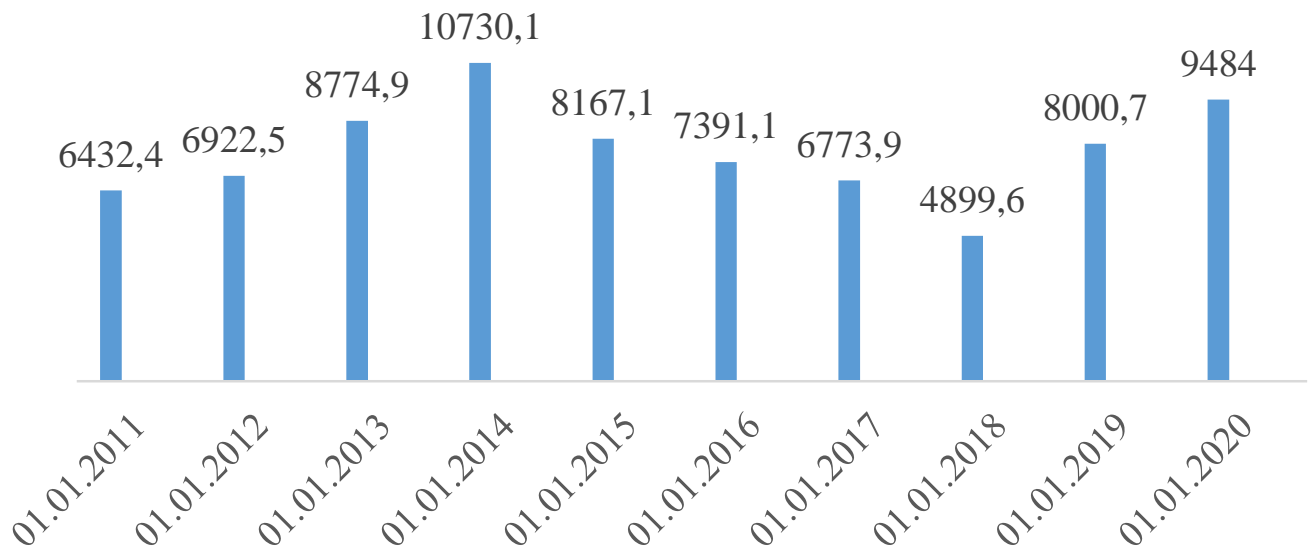

Рисунок 3 - Динамика объема утилизаџии загрязняющих вещзеств, тысяч тонн

Примечание - Составлен на основе источника: Официальный ресурс: Данные РГП «КазГидроМет» //http://www.kazhydromet.kz

Серьезные проблемы, с которыми постоянно сталкиваются отрасли (в том числе и климатические особенности), а также их специфические особенности усиливают отставание предприятий в области применения современных инструментов управления. В настоящее время в окружающей среде в достаточной мере накоплены потенциально опасные химические вещества, которые образовались в результате работы крупных промышленных предприятий, добычи и использования полезных ископаемых.

Об этом в своих исследованиях Т. Alimbaev, Z. Mazhitova, C. Beksultanova, N. Tentigul Kyzy (2020) рассматривают вопросы, связанные с современными экологическими проблемами, возникшими в связи с деятельностью предприятий горно-металлургической промышленности Республики Казахстан, которые подчеркивают, что в связи с промышленным прогрессом горнодобывающей и металлургической промышленности, которая является одним из основных секторов республиканской экономики, уровень загрязнения окружающей среды возрастает.

Авторы на примерах доказывают, что, с одной стороны, рост экономического потенциала горнометаллургической промышленности и переход к рыночным механизмам развития экономики привели к значительному увеличению потенциала республики как крупного экспортера и лидера среди мировых сырьевых держав. С другой стороны, рост промышленного производства привел к реальной угрозе возникновения экологического кризиса в республике. Авторы пришли к выводу, что возможно решить экологические проблемы путем совершенствования мер по охране атмосферного воздуха и повышения эффективности использования и охраны водных ресурсов. Кроме того, уменьшение химической нагрузки на почву, усиление работы по охране, воспроизводству и рациональному использованию растительного и животного мира, внедрение очистных сооружений и установок, особенно в местах добычи полезных ископаемых, создадут благоприятные благоприятные условия для значительного улучшения экологической ситуации в регионе [25].

Изучая опыт, который был исследован казахстанскими учеными в отношении качества экономического пространства с выявлением значения фактора экономического пространства для разработки рекомендаций по улучшению его качества в урбанизированных пространствах агломераций (Притворова Т.П., Куттыбаева Н.Б., 2016), нами был применен индексный метод анализа конкурентоспособности с учетом структурных сдвигов и конкурентоспособности местных производителей, который позволяет оценить динамику конкурентоспособности отрасли региона в целом [26].

Примененный нами метод анализа конкурентоспособности базовых и высокотехнологичных отраслей Центрального Казахстана с применением метода структурно-динамического анализа позволяет оценить конкурентоспособность отрасли.

Анализ полученных результатов оценки STR-эффекта и KON-эффекта по базовым и высокотехнологичным отраслям для каждого из регионов Центрального Казахстана за период с 2014-2019 годы и их динамика представлены в таблицах 2, 3, 4 .

Таблица 2

Результаты оценки STR-эффекта и KON-эффекта по базовым и высокотехнологичным отраслям для Карагандинской области за период с 2014-2019 годы

\begin{tabular}{|c|c|c|c|c|c|}
\hline \multirow{2}{*}{ Год } & \multicolumn{2}{|c|}{ Базисный показатель } & \multicolumn{2}{|c|}{ Цепной показатель } \\
\cline { 2 - 3 } & STR-эффект & KON -эффект & STR-эффект & КОN -эффект \\
\hline \multicolumn{3}{|c|}{ Горнодобывающая промышленность } \\
\hline 2014 & $-0,0735$ & 6,1256 & 2014 & $-0,0735$ & 6,1256 \\
\hline
\end{tabular}




\begin{tabular}{|c|c|c|c|c|c|}
\hline 2015 & $-1,9606$ & 1,7894 & 2015 & $-2,4514$ & $-2,1799$ \\
\hline 2016 & $-2,7532$ & 2,9636 & 2016 & $-0,2798$ & 0,6805 \\
\hline 2017 & $-2,8710$ & 5,9162 & 2017 & 0,3573 & 1,5426 \\
\hline 2018 & $-1,9794$ & 6,4702 & 2018 & 1,1381 & $-0,6501$ \\
\hline 2019 & -22472 & 8,3800 & 2019 & $-0,0766$ & 0,7523 \\
\hline \multicolumn{6}{|c|}{ Химическая промышленность } \\
\hline 2014 & 0,3271 & 0,5430 & 2014 & 0,3271 & 0,5430 \\
\hline 2015 & 0,7575 & 0,5564 & 2015 & 0,5742 & $-0,0121$ \\
\hline 2016 & 0,7597 & 1,1921 & 2016 & $-0,2424$ & 0,5020 \\
\hline 2017 & 0,8438 & 1,3585 & 2017 & $-0,0664$ & $-0,0258$ \\
\hline 2018 & 1,0347 & 0,6618 & 2018 & 0,0225 & $-0,5560$ \\
\hline 2019 & 1,4885 & 0,2731 & 2019 & 0,2319 & $-0,2686$ \\
\hline \multicolumn{6}{|c|}{ Металлургическая промышленность } \\
\hline 2014 & 2,6888 & $-6,5337$ & 2014 & 2,6888 & $-6,5337$ \\
\hline 2015 & 19,6593 & $-5,7733$ & 2015 & 14,1708 & 7,2417 \\
\hline 2016 & 44,1260 & $-14,2790$ & 2016 & 16,1890 & $-42,7615$ \\
\hline 2017 & 53,6421 & $-18,6506$ & 2017 & 0,4586 & $-9,0677$ \\
\hline 2018 & 56,6830 & $-24,8293$ & 2018 & $-3,5567$ & $-17,0841$ \\
\hline 2019 & 60,7170 & $-35,5236$ & 2019 & $-0,2012$ & $-32,8660$ \\
\hline \multicolumn{6}{|c|}{ ІФармацевтическая промышленность } \\
\hline 2014 & 0,0001 & 0,0065 & 2014 & 0,0001 & 0,0065 \\
\hline 2015 & 0,0072 & 0,0642 & 2015 & 0,0075 & 0,0531 \\
\hline 2016 & 0,0092 & 0,0309 & 2016 & $-0,00004$ & $-0,0476$ \\
\hline 2017 & 0,0281 & 0,0433 & 2017 & 0,0186 & $-0,0027$ \\
\hline 2018 & 0,0249 & 0,1893 & 2018 & $-0,0077$ & 0,0813 \\
\hline 2019 & 0,0352 & 0,2617 & 2019 & 0,0141 & 0,0204 \\
\hline \multicolumn{6}{|c|}{ Сфера IT-услуг (производство компьютеров, электронной и оптической продукции, услуг) } \\
\hline 2014 & 0,0103 & 0,0044 & 2014 & 0,0103 & 0,0044 \\
\hline 2015 & 0,0221 & 0,0116 & 2015 & 0,0131 & 0,0070 \\
\hline 2016 & 0,0116 & 0,0327 & 2016 & $-0,0169$ & 0,0181 \\
\hline 2017 & $-0,0212$ & 0,4354 & 2017 & $-0,0290$ & 0,2745 \\
\hline 2018 & $-0,0708$ & $-0,0210$ & 2018 & $-0,1016$ & $-0,2335$ \\
\hline 2019 & $-0,0409$ & $-0,0900$ & 2019 & 0,0157 & $-0,0324$ \\
\hline
\end{tabular}

Источник: Составлена автором на основе расчетов

Таблица 3

Результаты оценки STR-эффекта и KON-эффекта по базовым и высокотехнологичным отраслям для Павлодарской области за период с 2014-2019 годы

\begin{tabular}{|c|c|c|c|c|c|}
\hline \multirow{2}{*}{ Год } & \multicolumn{2}{|c|}{ Базисный показатель } & \multirow{2}{*}{ Год } & \multicolumn{2}{|c|}{ Цепной показатель } \\
\hline & STR-эффект & KON -эффект & & STR-эффект & KON -эффект \\
\hline \multicolumn{6}{|c|}{ Горнодобывающая промышленность } \\
\hline 2014 & $-0,0394$ & 0,3688 & 2014 & $-0,0394$ & 0,3688 \\
\hline 2015 & $-1,0527$ & 3,4158 & 2015 & $-1,2827$ & 3,8038 \\
\hline 2016 & $-1,4782$ & 8,2932 & 2016 & $-0,2831$ & 5,1452 \\
\hline 2017 & $-1,5414$ & 17,0180 & 2017 & 0,4917 & 6,6318 \\
\hline 2018 & $-1,0627$ & 17,2720 & 2018 & 1,7550 & $-3,4619$ \\
\hline 2019 & $-1,2065$ & 19,8116 & 2019 & $-0,1030$ & 0,8482 \\
\hline \multicolumn{6}{|c|}{ Химическая промышленность } \\
\hline 2014 & 0,1788 & $-0,1047$ & 2014 & 0,1788 & $-0,1047$ \\
\hline 2015 & 0,4141 & $-0,0801$ & 2015 & 0,2939 & 0,0357 \\
\hline 2016 & 0,4154 & $-0,0720$ & 2016 & $-0,1337$ & 0,0286 \\
\hline 2017 & 0,4613 & 0,0813 & 2017 & $-0,0334$ & 0,1613 \\
\hline 2018 & 0,5657 & 0,3342 & 2018 & 0,0116 & 0,1774 \\
\hline 2019 & 0,8138 & 0,6540 & 2019 & 0,1602 & 0,1736 \\
\hline \multicolumn{6}{|c|}{ Металлургическая промышленность } \\
\hline 2014 & 1,4542 & 1,3604 & 2014 & 1,4542 & 1,3604 \\
\hline
\end{tabular}




\begin{tabular}{|l|l|l|l|l|l|}
\hline 2015 & 10,6329 & $-0,8444$ & 2015 & 11,8826 & $-2,8444$ \\
\hline 2016 & 23,8658 & $-5,7857$ & 2016 & 12,8575 & $-5,6906$ \\
\hline 2017 & 29,0127 & $-3,7854$ & 2017 & 0,3702 & 3,1079 \\
\hline 2018 & 30,6574 & $-6,2224$ & 2018 & $-2,8201$ & $-1,4532$ \\
\hline 2019 & 32,8392 & $-13,8723$ & 2019 & $-0,1560$ & $-4,8262$ \\
\hline \multicolumn{7}{|c|}{ Фармацевтическая промышленность } \\
\hline 2014 & 0,0002 & $-0,0427$ & 2014 & 0,0002 & $-0,0427$ \\
\hline 2015 & 0,0152 & $-0,0375$ & 2015 & 0,0092 & 0,0051 \\
\hline 2016 & 0,0193 & $-0,0682$ & 2016 & 0,0000 & $-0,0261$ \\
\hline 2017 & 0,0593 & $-0,1205$ & 2017 & 0,0131 & $-0,0157$ \\
\hline 2018 & 0,0524 & $-0,1351$ & 2018 & $-0,0038$ & $-0,0034$ \\
\hline 2019 & 0,0742 & $-0,1626$ & 2019 & 0,0030 & $-0,0024$ \\
\hline 2014 & & Cфера IT-услуг & \multicolumn{3}{c|}{} \\
\hline 2015 & 0,0002 & 0,0008 & 2014 & 0,0002 & 0,0008 \\
\hline 2016 & 0,0004 & 0,0003 & 2015 & 0,0004 & $-0,0005$ \\
\hline 2017 & 0,0002 & 0,0540 & 2016 & $-0,0004$ & 0,0686 \\
\hline 2018 & $-0,0004$ & 0,0181 & 2017 & $-0,0126$ & $-0,0334$ \\
\hline 2019 & $-0,0013$ & $-0,0025$ & 2018 & $-0,0048$ & $-0,0140$ \\
\hline
\end{tabular}

Источник: Составлена автором на основе расчетов 
Таблица 4

Результаты оценки STR-эффекта и KON-эффекта по базовым и высокотехнологичным отраслям для ВКО за период с 2014-2019 годы

\begin{tabular}{|c|c|c|c|c|c|}
\hline \multirow{2}{*}{ Год } & \multicolumn{2}{|c|}{ Базисный показатель } & \multirow{2}{*}{ Год } & \multicolumn{2}{|c|}{ Цепной показатель } \\
\hline & STR-эффект & KON -эффект & & STR-эффект & KON -эффект \\
\hline \multicolumn{6}{|c|}{ Горнодобывающая промышленность } \\
\hline 2014 & $-0,0485$ & $-0,4792$ & 2014 & $-0,0485$ & 0,4792 \\
\hline 2015 & $-1,2956$ & 3,5120 & 2015 & $-1,1788$ & 2,8521 \\
\hline 2016 & $-1,8194$ & 3,6355 & 2016 & $-0,2497$ & $-0,7357$ \\
\hline 2017 & $-1,8972$ & 7,8490 & 2017 & 0,2671 & 2,2363 \\
\hline 2018 & $-1,3080$ & 18,4935 & 2018 & 1,0592 & 5,3043 \\
\hline 2019 & $-1,4850$ & 33,7541 & 2019 & $-0,0932$ & 7,4587 \\
\hline \multicolumn{6}{|c|}{ Химическая промышленность } \\
\hline 2014 & 0,3442 & 0,3079 & 2014 & 0,3442 & 0,3079 \\
\hline 2015 & 0,7971 & $-0,1104$ & 2015 & 0,5323 & $-0,3880$ \\
\hline 2016 & 0,7995 & $-0,5508$ & 2016 & $-0,2010$ & $-0,4113$ \\
\hline 2017 & 0,8879 & $-1,1203$ & 2017 & $-0,0366$ & $-0,3149$ \\
\hline 2018 & 1,0888 & $-1,2623$ & 2018 & 0,0114 & 0,0543 \\
\hline 2019 & 1,5663 & $-1,6768$ & 2019 & 0,1388 & $-0,0975$ \\
\hline \multicolumn{6}{|c|}{ Металлургическая промышленность } \\
\hline 2014 & 2,2956 & 1,7301 & 2014 & 2,2956 & 1,7301 \\
\hline 2015 & 16,7847 & $-1,8101$ & 2015 & 13,8545 & $-35,7899$ \\
\hline 2016 & 37,6739 & 8,7715 & 2016 & 15,3777 & 112,8751 \\
\hline 2017 & 45,7986 & $-7,9288$ & 2017 & 0,4925 & $-152,6607$ \\
\hline 2018 & 48,3948 & $-15,6906$ & 2018 & $-3,6690$ & $-36,6911$ \\
\hline 2019 & 51,8390 & $-19,5517$ & 2019 & $-0,1857$ & $-8,3599$ \\
\hline \multicolumn{6}{|c|}{ ІФармацевтическая промышленность } \\
\hline 2014 & 0,0001 & 0,0086 & 2014 & 0,0001 & 0,0086 \\
\hline 2015 & 0,0095 & 0,0851 & 2015 & 0,0098 & 0,0687 \\
\hline 2016 & 0,0121 & 0,0409 & 2016 & $-0,00005$ & $-0,0660$ \\
\hline 2017 & 0,0373 & 0,0574 & 2017 & 0,0241 & $-0,0035$ \\
\hline 2018 & 0,0330 & 0,2511 & 2018 & $-0,0113$ & 0,1192 \\
\hline 2019 & 0,0467 & 0,0136 & 2019 & 0,0191 & $-0,1514$ \\
\hline \multicolumn{6}{|c|}{ Сфера IT-услуг } \\
\hline 2014 & 0,0017 & $-0,0056$ & 2014 & 0,0017 & $-0,0056$ \\
\hline 2015 & 0,0036 & 0,0197 & 2015 & 0,0015 & 0,0222 \\
\hline 2016 & 0,0019 & 0,0056 & 2016 & $-0,0050$ & $-0,0167$ \\
\hline 2017 & $-0,0034$ & 0,0019 & 2017 & $-0,0046$ & $-0,0024$ \\
\hline 2018 & $-0,0115$ & $-0,0019$ & 2018 & $-0,0050$ & $-0,0023$ \\
\hline 2019 & $-0,0066$ & $-0,0060$ & 2019 & 0,0028 & $\begin{array}{l}-0,0019 \\
\end{array}$ \\
\hline
\end{tabular}

Источник: Составлена автором на основе расчетов

Результаты исследования.

- $\quad$ все значения по оси X (STR - эффект) положительны, это означает в рамках методологии STR эффекта, что темп роста объема произведенной продукции в отрасли выше, чем темп роста объема произведенной продукции на национальном уровне;

- $\quad$ среди Y (KON-эффект) есть отрицательные значения, что означает, что темпы роста отрасли в конкретном регионе могут быть и выше и ниже национального отраслевого уровня;

- чем меньше значение $\mathrm{X}$ и оно ближе к оси $\mathrm{Y}$, тем менее значима отрасль в экономике региона. Точка, соответствующая Республике Казахстан в целом, находится в координатах $(1.1 ; 0)$. В этой точке X отражает разницу между отраслевым и национальным темпом роста, а Ү равно 0, т.к. для страны эти темпы роста совпадают. Во всех областях, находящихся справа от этой точки отрасль является значимой для экономики страны, т.к. её удельный вес в экономике региона выше, чем в целом по стране.

- $\quad$ для всех областей находящихся справа от точки, соответствующей Республике Казахстан и вертикальной оси, проведенной через эту точку можно сделать вывод о достаточной конкурентоспособности отрасли в масштабах страны и на внешних рынках. В то же время области с положительным значением Y находятся в стадии расширения объема произведенной продукции в пределах рассматриваемого промежутка 
времени с 2014 по 2019гг. а, следовательно, спрос на их продукцию есть на мировом и внутреннем рынке. Для всех областей с отрицательными значениями Y, имеет место сокращение объема произведенной продукции, т.к. происходит падение спроса на их продукцию.

На основе оценки конкурентоспособности базовых и высокотехнологичных отраслей Центрального Казахстана с применением метода структурно-динамического анализа была определена модель конкурентоспособности регионов, которая позволяет нам сделать вывод, что самыми стабильными регионами Центрального Казахстана с более равномерно развивающимися отраслями (к ним мы отнесли отрасли-лидеры и динамичные отрасли) являются:

1 Карагандинская область.

2 Павлодарская область.

3 Восточно-Казахстанская область.

Сводная информация по всем областям Центрального Казахстана представлена в (Таблица 5).

Таблица 5

Модель конкурентоспособности регионов Центрального Казахстана на основе метода структурнодинамического анализа

\begin{tabular}{|c|c|c|c|c|c|}
\hline Область & $\begin{array}{c}\text { Горнодо } \\
\text { бывающая }\end{array}$ & $\begin{array}{c}\text { Металлур } \\
\text { гическая }\end{array}$ & $\begin{array}{c}\text { Хими } \\
\text { ческая }\end{array}$ & $\begin{array}{c}\text { Фармацев } \\
\text { тическая }\end{array}$ & $\begin{array}{l}\text { Сфера IT- } \\
\text { услуг }\end{array}$ \\
\hline Карагандинская & B & $\mathrm{C}$ & A & A & A \\
\hline Павлодарская & $\mathrm{A}$ & B & $\mathrm{A}$ & B & $\mathrm{C}$ \\
\hline $\begin{array}{l}\text { Восточно- } \\
\text { Казахстанская }\end{array}$ & A & A & B & B & Д \\
\hline \multicolumn{6}{|c|}{$\begin{array}{l}\text { Отрасли лидеры с выходом на мировые рынки - ; д } \\
\text { состоянии кризиса или стагнации - ; отрасли аутсайдеры - }\end{array}$} \\
\hline
\end{tabular}

Для Карагандинской области модель региональной конкурентспособности является гораздо более проблемной. В то же время, такие сложившиеся в предшествующие исторические периоды агломерации, как Карагандинская городская агломерация, по всей видимости находится в стадии конвергенции, когда отдача от отрасли специализации (металлургии) убывает и она имеет темпы роста меньшие, чем среднеотраслевые темпы роста. Одновременно положительного перелива в форме эффекта роста в связанных экономических секторах (металлообработка, машиностроение) не наблюдается, что говорит об отсутствии эффекта урбанизации, под которым понимается возрастающая отдача от комплиментарных отраслей (кластеров) на уровне региона в целом. Кластеры являются воплощением этой комплиментарности и определяют рост региона в целом.

Для реализации и дальнейшего развития базовых отраслей необходима система мер государственной поддержки, которые рассмотрены для каждой отрасли. Проведенный анализ критериев выбора приоритетных для Центрального Казахстана базовых отраслей позволяет определить цели и задачи исследуемых отраслей.

Обследование практики работы многих компаний и анализ теоретических исследований, проведенный нами, показал, что формирование бизнес-модели позволяет на достаточно конструктивной основе совершенствовать традиционную систему управления предприятием единичного типа, однако требует теоретического обоснования выбора характеристик и свойств управления с учетом отраслевых особенностей предприятия, его специализации

Для реализации данных проектов и их дальнейшего развития необходима система мер государственной поддержки отраслей, которые были рассмотрены для каждой отрасли.

\section{Выводы}

Рассмотренные нами концепции региональной конкурентоспособности представляют собой достаточно длительную традицию и отдельную ветку экономической науки, в которой методология базируется на таких системных свойствах региона как его ограниченность, автономность, относительная однородность и связанность. Теории региональной конкурентоспособности в современной экономической науке представляют диверсифицированную отрасль знания. Внутри этого направления можно выделить неоклассические модели, которые при всех своих достоинствах не учитывают факторы экономического пространства.

С точки зрения включения в модели характеристик и объектов экономического пространства можно выделить новую экономическую географию и теорию международной торговли, которые учитывают формы и тенденции размещения деятельности в пространстве и экономические связи в условиях разных пространственных структур.

Теории и модели региональной экономики создаются для того, чтобы объяснить наблюдаемые процессы размещения хозяйствующих субъектов в экономическом пространстве, выявить тенденции и факторы конкурентоспособности отдельных территорий, объяснить различия между ними в уровне экономического развития и спрогнозировать тренды ближайшего будущего. 
Систематизация методов оценки конкурентоспособности регионов позволила сделать вывод, что ни один из используемых методов не основан на признании количественной взаимосвязи между параметрами экономического пространства (плотность, размещение, связанность) и экономическим ростом отраслей и не позволяет установить такую взаимосвязь в функциональной, либо корреляционной форме.

В связи с этим, нами была поставлена и решена задача установления такой взаимосвязи по результатам применения сложного индекса, известного как индекс структурно-динамического анализа, который известен в зарубежной литературе как инструмент «shift-share analysis», но понимание его затруднено не только сложностью расчетов, но и трудностями объяснения.

В связи с этим, нами была предпринята попытка упрощения расчетов без потери содержания и представления их результатов в графической форме для облегчения трактовки. В результате факторы, определяющие региональное развитие под влиянием спроса на продукцию отрасли в целом - STR эффект, a конкурентоспособность местного производства по сравнению с национальным отраслевым уровнем - KON эффект.

Используемый нами индексный метод анализа конкурентоспособности на основе структурных сдвигов в отраслях позволил оценить конкурентоспособность каждой отрасли региона. Введение структурных компонентов, т.е. рассмотрение отрасли с учетом структурных сдвигов в экономике региона и страны позволяет дать оценку факторам роста экономики региона. В результате расчетов STR -эффекта и KON - эффекта был рассчитан абсолютный прирост STR-эффекта и KON-эффекта по базовым и высокотехнологичным отраслям Центрального Казахстана и показана модель конкурентоспособности региона на основе метода структурнодинамического анализа.

Проведенная с помощью этих показателей региональная диагностика позволяет выявить модель его конкурентоспособности: опорные, динамичные, кризисные и аутсайдерские отрасли. Анализ моделей конкурентоспособности позволил нам сделать вывод, что самыми стабильными регионами с более равномерно развивающимися отраслями (к ним мы отнесли отрасли-лидеры и динамичные отрасли) являются:

1 Карагандинская область.

2 Павлодарская область.

3 Восточно-Казахстанская область.

Результат анализа технико-технологических сдвигов и качества выпускаемой продукции в процессе реализации проектов в базовых отраслях и отраслях «высоких технологий», показал, что необходим:

1) Переход базовых отраслей на более высокий технико-технологический уровень: рост доли прогрессивных и уникальных технологий, обеспечивающих высокий уровень производительности труда и ресурсосбережение;

2) Вертикальная и горизонтальная диверсификация выпускаемой продукции в базовых отраслях.

3) Усиление конкурентных позиций продукции базовых отраслей.

4) Достижение экологического эффекта: сокращение вредных выбросов и техногенных отходов.

На основе анализа инновационного развития регионов Центрального Казахстана (Карагандинская, Павлодарская, ВКО области) были показаны факторы, влияющие на активизацию инновационного предпринимательства и выделение наиболее перспективных отраслей с целью систематизации мер по их дальнейшему совершенствованию, поддержки и повышению инновационной активности.

Одним из показателей, с помощью которого можно судить об инновационной активности региона является объем инновационной продукции. На данный показатель оказывают влияние факторы разного характера, в том числе экономические и научно-технологические.

Проведенный анализ критериев выбора приоритетных для Центрального Казахстана базовых отраслей позволил определить цели и задачи исследуемых отраслей, которые направлены на:

- внедрение прогрессивных и уникальных технологий (восстановление традиционных производств, поддержка НИОКР);

- производство продукции с более высокой добавленной стоимостью (создание новых высокопроизводительных предприятий);

- экспортная ориентация и повышение казахстанского содержания.

С учетом экспертной оценки, проведенной в форме открытого интервью с представителями местного бизнессообщества и органов местного управления, нами было выявлено, что для реализации данных проектов и их дальнейшего развития необходима система мер государственной поддержки для каждой из отраслей, на основе чего была разработана стратегическая карта целей и задач по развитию базовых отраслей Центрального Казахстана. В процессе исследования были выявлены основные направления развития каждой из отраслей, где за основу были рассмотрены базовые предприятия, реализующие или готовые к реализации проектов. Для каждой отрасли были рассмотрены показатели развития отраслей и особенности внедрения инновационных методов управления.

Таким образом, реализация намеченных проектов позволит увеличить диверсификацию экономики регионов и создать конкурентоспособное экономическое пространство, которое позволит в полной мере извлечь агломерационные эффекты. 


\section{Список литературы:}

1 S.A. Bogatenkov, V.A. Belevitin, M.L. Khasanova Risk Management Based on Model of Competences when Introducing Innovative Information Technology // International Journal of Engineering \& Technology 7 (4.38) (2018) 78$81 \mathrm{https} / / / \mathrm{www}$. researchgate.net/deref/http\%3A\%2F\%2Fwww.sciencepubco.com\%2Findex.php\%2FIJET

2 Novichkov, N. V., Novichkova, A. V., \& Malygina, O. V. (2018). Tourism business innovation in the experience economy. Servis v Rossii i za rubezhom [Services in Russia and Abroad], 12(2), 6-19. doi: 10.24411/1995- 042X-201810201.

3 А.С. Кулмаганбетова, И.Н. Дубина, Ж.Б. Рахметулина Покомпонентная оценка инновационного потенциала субъектов малого и среднего бизнеса: опыт Казахстана //Экономика. Профессия. Бизнес. 2019 №3. c.57. DOI: $10.14258 / \mathrm{epb} 201934$

4 Bell, R. (2015). Developing the next generation of entrepreneurs: Giving students the opportunity to gain experience and thrive. International Journal of Management Education, 13(1), p.37-47 https://doi.org/10.1016/j.ijme.2014.12.002.

5 Karimi, S., Biemans, H. J. A., Mahdei, K. N., Lans, T., Chizari, M., \& Mulder, M. (2015). Testing the relationship between personality intentions in a developing country. International Journal of Psychology, 1-15. https://doi.org/10.1002/ijop

6 Prakash, D., Jain, S., \& Chauhan, K. (2015). Entrepreneurial intensity in relation to presence of entrepreneurship development cell: A study of institutes offering professional courses in national capital region Delhi. International Journal of Management Education, 13(1), 95-105 https://doi.org/10.1016/j.ijme.2015.01.004

7 Moroz, P. W., \& Hindle, K. (2012). Entrepreneurship as a process: Toward harmonizing multiple perspectives. Entrepreneurship: Theory and Practice, 36(4), 781-818 https://doi.org/10.1111/j.1540-6520.2011.00452.x

8 Solesvik, M. Z., Westhead, P., Matlay, H., \& Parsyak, V. N. (2013). Entrepreneurial assets and mindsets benefit from university entrepreneurship education investment, 748-762. https://doi.org/10.1108/ET-06-2013-0075

9 Bobylev S.N., Solovyeva S. V. (2020) Circular economy and its indicators for Russia. Mir novoi ekonomiki. The World of the New Economy. - 14(2). p.63-72. DOI: 10.26794/2220-6469-2020-14-2-63-72

10 Ayman Abu-Rumman, Ata Al Shraah, Faisal Al-Madi, Tasneem Alfalah (2021) Entrepreneurial networks, entrepreneurial orientation, and performance of small and medium enterprises: are dynamic capabilities the missing link? // Journal of Innovation and Entrepreneurship 10:29. https://doi.org/10.1186/s13731-021-00170-8

11 Abriham Ebabu Engidaw (2021) Exploring internal business factors and their impact on firm performance: small business perspective in Ethiopia // Journal of Innovation and Entrepreneurship 10:25. https://doi.org/10.1186/s13731-02100167-3

12 Takawira Munyaradzi Ndofirepi (2020) Relationship between entrepreneurship education and entrepreneurial goal intentions: psychological traits as mediators // Journal of Innovation and Entrepreneurship. 9:2. https://doi.org/10.1186/s13731-020-0115-x

13 Nieves J., Segarra-Cipres M. Management innovation in the hotel industry //Tourism Management. 2015. V.46. P.51-58. http://www.sciencedirect.com/science/article/pii/S0261517714001046. DOI: 10.1016/j.tourman.2014.06.002

14 Novichkov, N. V., Novichkova, A. V., \& Malygina, O. V. (2018). Tourism business innovation in the experience economy. Servis v Rossii i za rubezhom [Services in Russia and Abroad], 12(2), 6-19. doi: 10.24411/1995- 042X-201810201.

15 Орлов С.Н. Проблемы развития малого и среднего бизнеса в условиях динамичного развития //Управление в современных системах №1(25) 2020. с.27 ISSN 2311-1313 http://journal.inueco.ru/journal_25_4/

16 Kirilchuk, S. P., \& Nalivaychenko, E. V. (2017). The Development of Globality of Innovative Modifications. //Journal of Fundamental and Applied Sciences, 9(1S), p.1025-1048. doi: 10.4314/jfas.v9i1s.754.

17 Стратегический план Министерства финансов Республики Казахстан на 2017 - 2021 годы, утвержденный приказом Министра финансов Республики Казахстан от 20 декабря 2016 года № 674 с внесенными изменениями от 28 декабря 2017 года № 762

18 Ștefan Cristian Gherghina, Mihai Alexandru Botezatu, Alexandra Hosszu, Liliana Nicoleta Simionescu (2020) Small and Medium-Sized Enterprises (SMEs): The Engine of Economic Growth through Investments and Innovation // Sustainability. 12(1), p.347. https://doi.org/10.3390/su12010347

19 Чебуханова Л. В. Финансирование малых и средних инновационных предприятий в Великобритании // Вестник Бурятского государственного университета. Экономика и менеджмент. 2019. № 3. С. 78-86. DOI 10.18101/2304-4446-2019-3-78-86

20 Kazakova M.V., Pospelova E.A. (2017). Kachestvo infrastruktury kak odno iz ogranicheniy ekonomicheskogo rosta: sravnitelnyy analiz Rossii i stran mira [The quality of infrastructure as one of the limitations of economic growth: a comparative analysis of Russia and countries of the world]. Journal of International Economic Affairs. 7 (3). doi: 10.18334/eo.7.3.38071.

21 Maschenko M.V., Volkova E.A. (2018). Vozdeystvie investitsionnyh protsessov na razvitie gornodobyvayuschikh predpriyatiy v Rossii [The impact of investment processes on the development of mining enterprises in Russia]. Journal of Economics, Entrepreneurship and Law. 8 (2). doi: 10.18334/epp.8.2.38800. 
22 Ovsyannikova T.Yu., Rabtsevich O.V., Yugova I.V. (2018). Disproportsii na rynke zhilischnyh investitsiy: analiz prichin i otsenka posledstviy [Imbalances in the housing investment: an analysis of the causes and assessing the consequences]. Russian Journal of Housing Research. 5 (4). doi: 10.18334/zhs.5.4.39694.

23 T. Alimbaev, Z. Mazhitova, C. Beksultanova, N. Tentigul Kyzy (2020) Activities of mining and metallurgical industry enterprises of the Republic of Kazakhstan: environmental problems and possible solutions // E3S Web of Conferences 175, 14019 (2020) INTERAGROMASH. https://doi.org/10.1051/e3sconf/202017514019

24 Официальный ресурс: Данные РГП «КазГидроМет» //http://www.kazhydromet.kz

25 Электронный ресурс: Данные Комитета по статистике Республики Казахстан за 2014-2019гг. //www.stat.gov.kz

26 Притворова Т.П., Куттыбаева Н.Б. Метод анализа региональной конкурентоспособности на основе структурных сдвигов в занятости населения //New approaches in economy and management: materials of the VI international scientific conference on September 15-16, 2016. Prague: Vědecko vydavatelské centrum «Sociosféra-CZ», 2016. 90 p. 